Supplement of Saf. Nucl. Waste Disposal, 1, 9-10, 2021

https://doi.org/10.5194/sand-1-9-2021-supplement

(C) Author(s) 2021. CC BY 4.0 License.

Supplement of

\title{
PREDIS: innovative ways for predisposal treatment and monitoring of low and medium radioactive waste
}

Ernst Niederleithinger et al.

Correspondence to: Ernst Niederleithinger (ernst.niederleithinger@bam.de)

The copyright of individual parts of the supplement might differ from the article licence. 
SafeND Symposium 2021

\section{PREDIS: INNOVATIVE WAYS FOR PRE-DISPOSAL} TREATMENT AND MONITORING OF LOW AND MEDIUM RADIOACTIVE WASTE

Ernst Niederleithinger ${ }^{1}$, Vera Lay ${ }^{1}$, Christian Köpp ${ }^{1}$, Erika Holt² ${ }^{2}$ Maria Oksa² ${ }^{1} \mathrm{BAM}{ }^{2} \mathrm{VTT}$ 


\section{Safety in technology and chemistry}
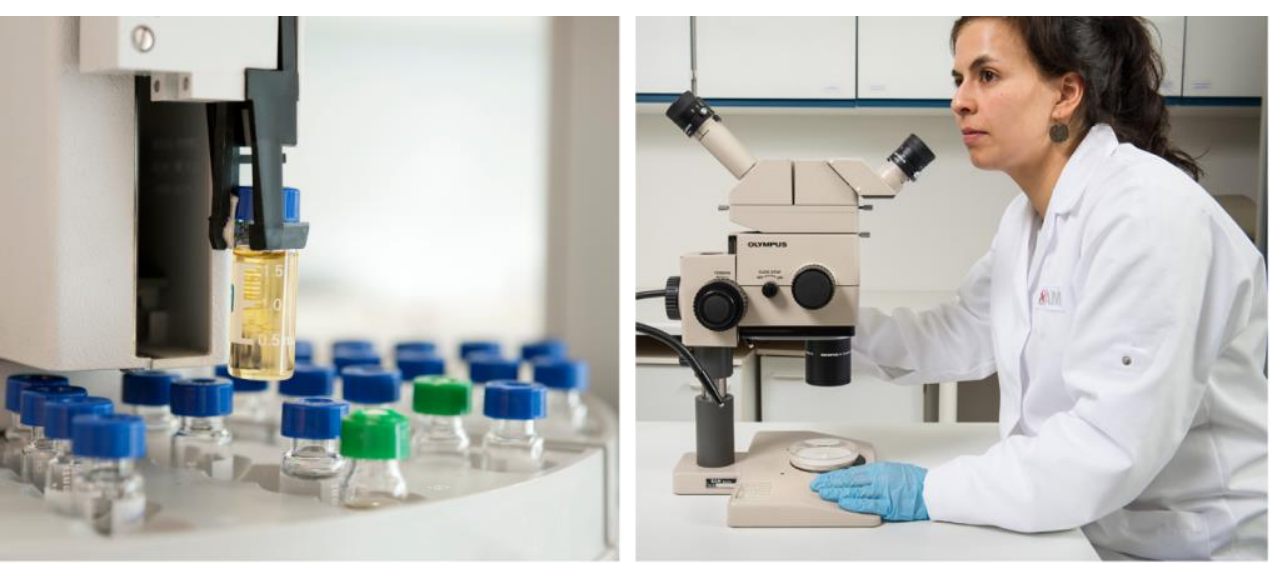

BAM is a senior scientific and technical federal authority with responsibility to the Federal Ministry for Economic Affairs and Energy (BMWi)
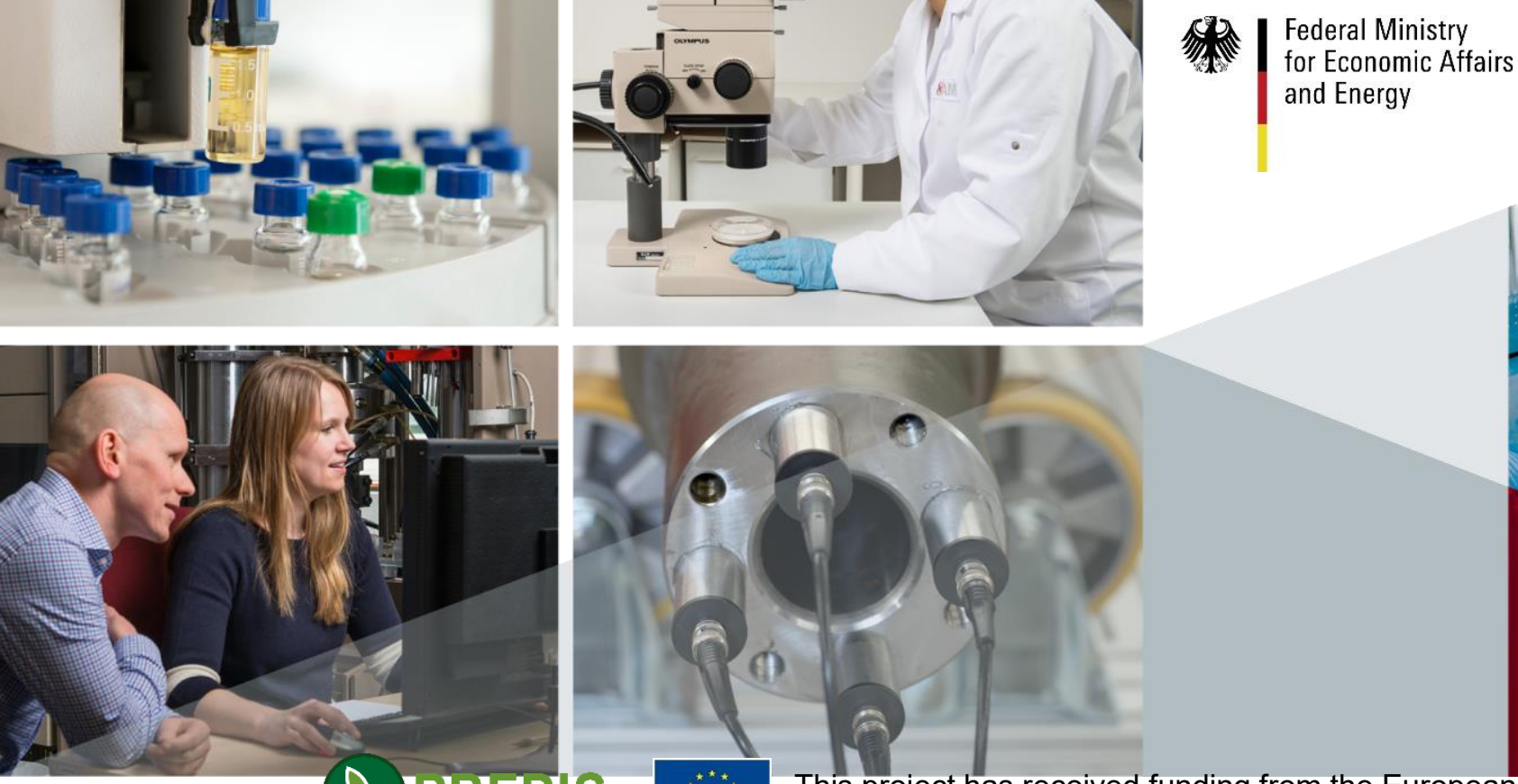


\section{Our tasks}

\section{Research, testing and consultation under one roof}

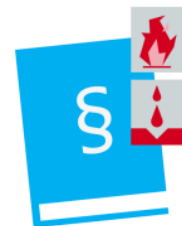

Statutory duties for technical safety

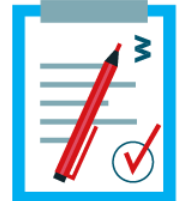

Consulting the German Federal Government and Industry

Research and development

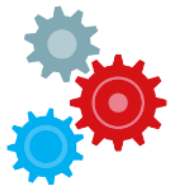

Contribution to standards and statutory regulations

Testing, analysis, approval

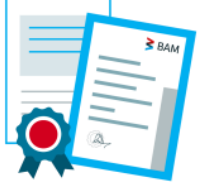




\section{One of five focus areas}

\section{Activities}

- Energy storage

- Energy conversion

- Energy transport

- Nuclear waste management
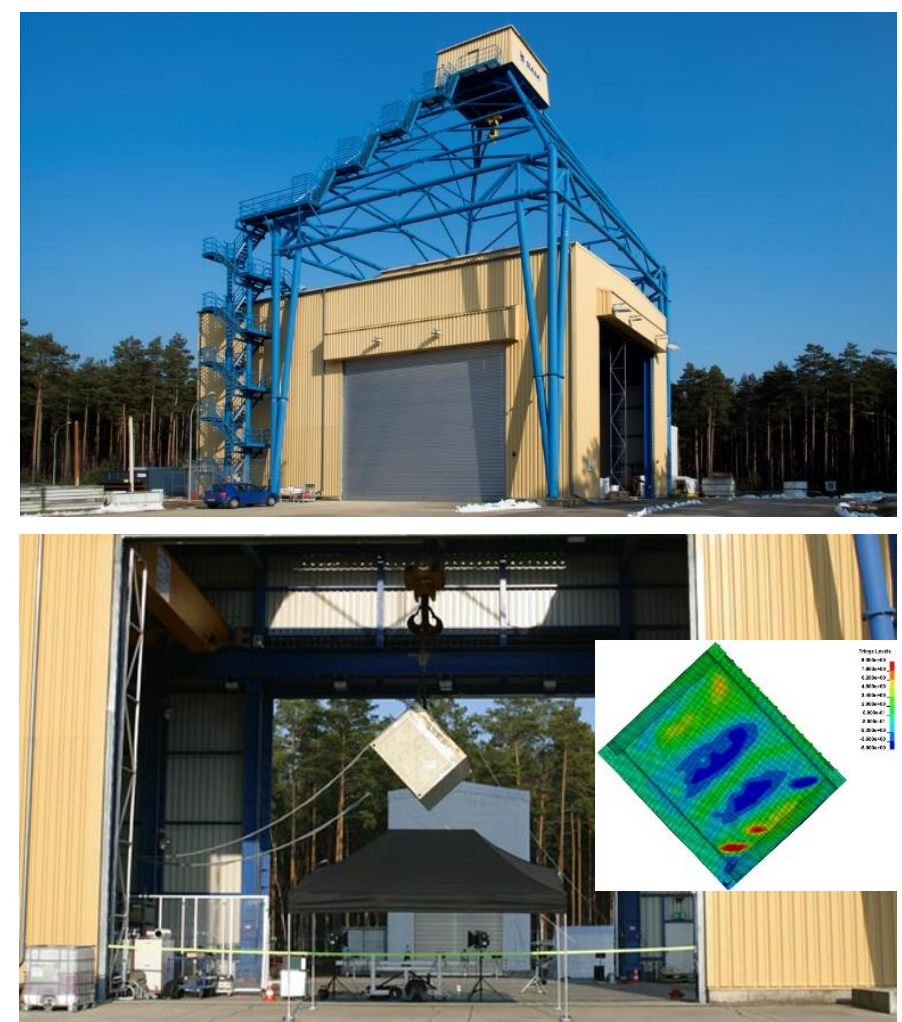


\section{BAM Division 8.2}

\section{NDT methods for civil engineering}

- Development, optimization and application of non-destructive testing methods for inspection an monitoring of all kinds of constructions

- Methods: Ultrasound, Radar, LIBS, embedded sensors

- Combination with competencies of other divisions (fiber optics, AE, NMR, CT/X-Ray, half cell potential:

We have it (almost) all! Automatisierung, Digitalisierung

- Application focus: Infrastructure \& buildings, new constructions methods and materials, nuclear constructions

- $\quad$ R \& D, validation, standardization, testing (special cases), trainings/workshops

Web: BAM-WWW-Link, contact: PD Dr. Ernst Niederleithinger

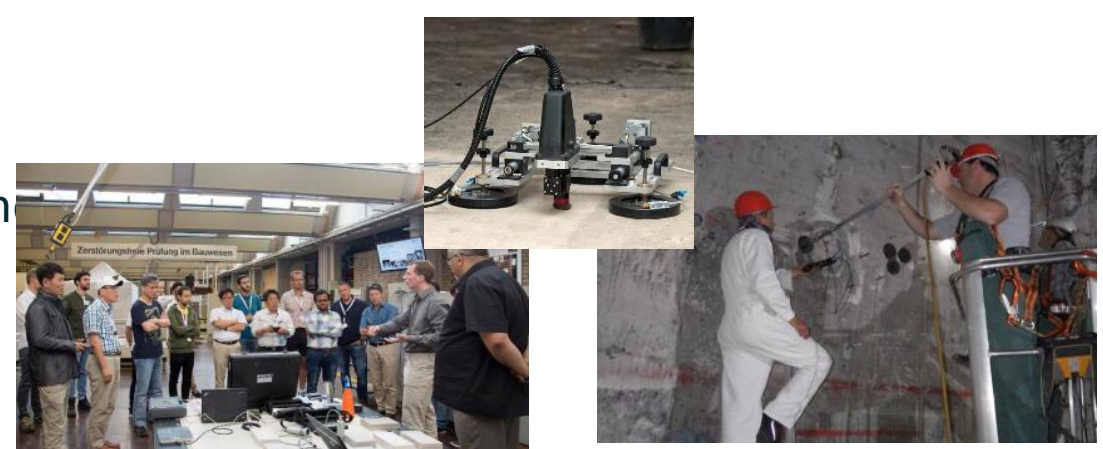




\section{Euratom PREDIS}

Low \& intermediate level waste types

EU-project PREDIS:

Pre-disposal management of radioactive waste

Coordination:

Erika Holt , Maria Oksa (VTT)

47 partners

24.7 M€ budget

https://predis-h2020.eu

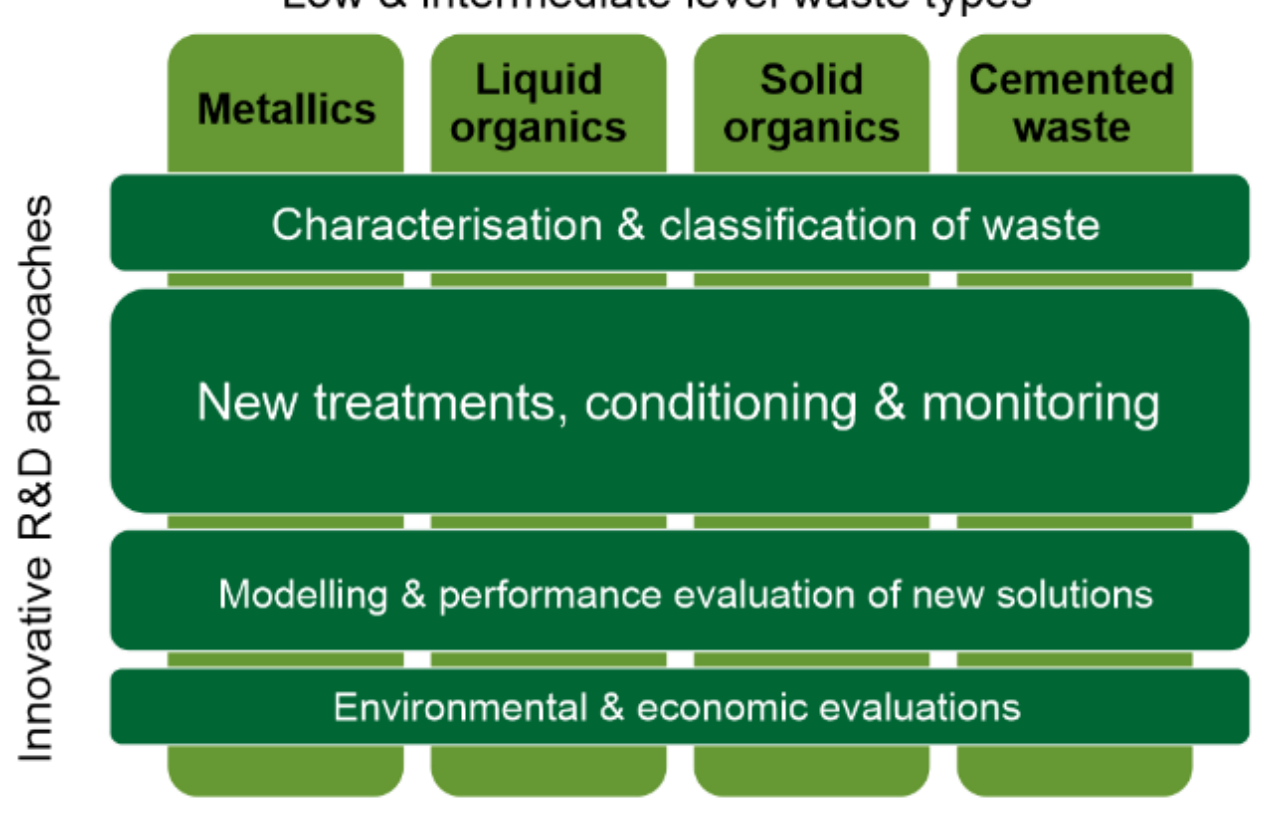




\section{Euratom PREDIS Objectives}

- PREDIS high-level, overall objectives are to:

Develop solutions (methods, processes, technologies and demonstrators) for future treatment and conditioning of waste across a number of MSs for which no industrially mature or inadequate solutions are currently available, improving safety during next waste management steps;

- or improve existing solutions with safer, cheaper or more effective alternative processes where they bring measurable benefits to several MSs (Member States).

- Analyse criteria, parameters and specifications for materials and packages with associated Waste Acceptance Criteria (WAC) for pre-disposal and disposal activities, supporting homogenisation of waste management processes across Europe.

These high-level objectives will be met by PREDIS having specific objectives:

1) Applying multi-disciplinary and multi-scale scientific approaches to demonstrate technical, economic and environmental feasibility of the new solutions;

2) Addressing project drivers from the end users' points-of-view;

3) Fostering deeper cooperation between experts from many EU Member-states and across generations;

4) Training new experts in the field of pre-disposal waste management technologies;

5) Updating and revising pre-disposal guiding documents (vision, SRA, roadmap, governance and deployment mechanisms), together with the EURAD EJP executive bodies. 


\section{Euratom PREDIS}

Where is the connection?

\section{Concrete!}
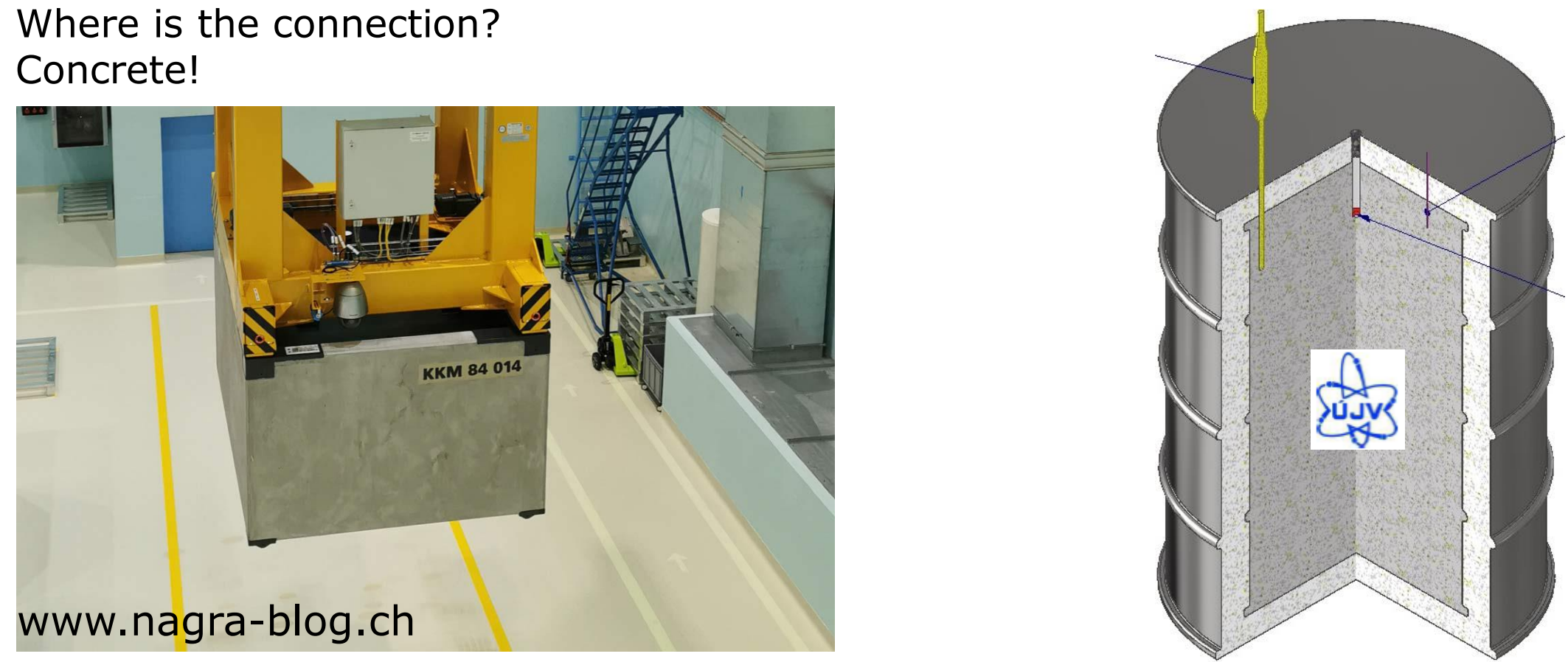


\section{Euratom PREDIS}

\section{WP7}

- Compile information about the state of the art of current methods and procedures for cemented waste management with specific focus on monitoring/long-term storage

- Identify, evaluate and demonstrate store and package quality assurance (mainly NDE) and monitoring technologies

- Adapt and demonstrate digital twin technology

- Develop and demonstrate methods for data handling incl. decision framework

- Identify opportunities for increased store automation, reducing human exposure to radiation

- Identify options for post treatment of packages and potential

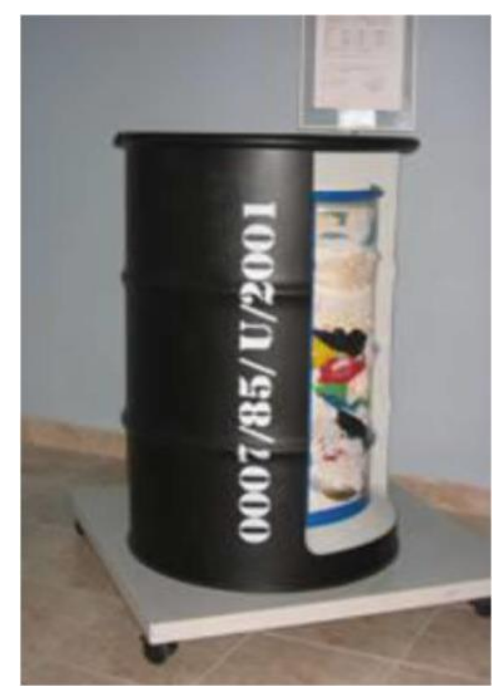
approaches to improve package design, construction and maintenance. 


\section{Euratom PREDIS}

\section{WP7}

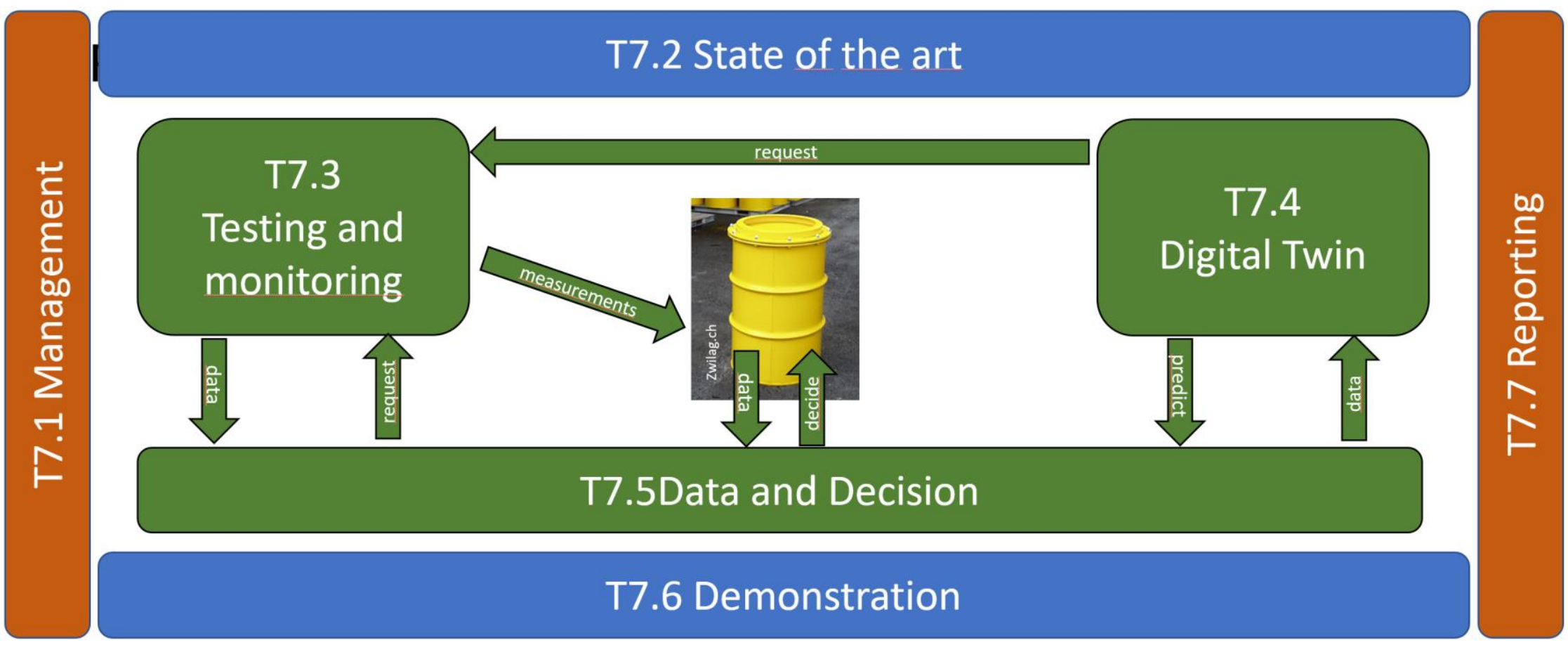




\section{Euratom PREDIS}

\section{WP7-T7.2: State of the Art}

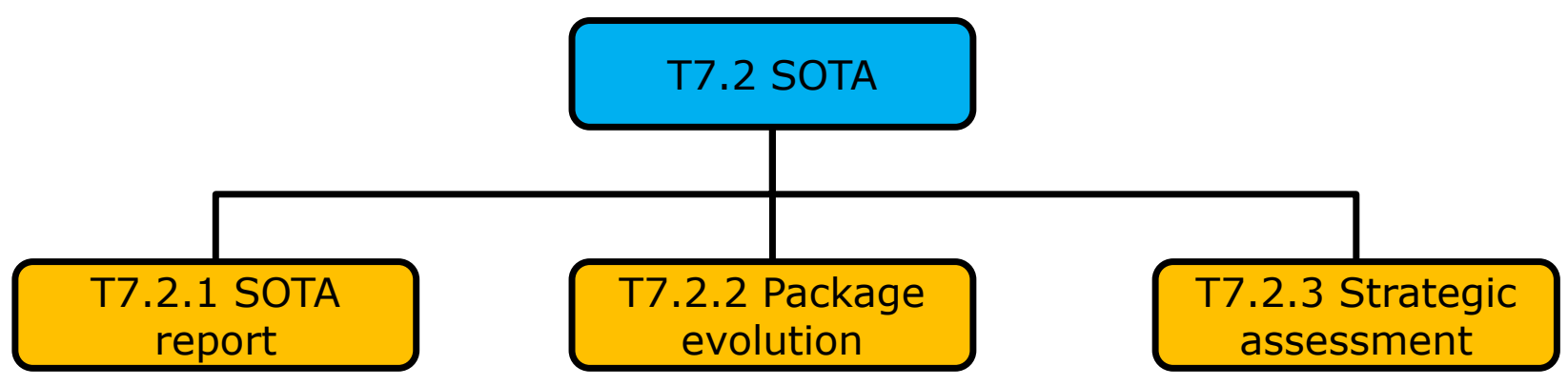

1st deliverable submitted: State of the art report:

https://predis-h2020.eu/wp-content/uploads/2021/03/PREDIS D7.1 WP7-SOTA V1-Final 2021 02.pdf 1st milestone accomplished:

Milestone 50 - Reference package and factors affecting package evolution and degradation (WP7, Task 7.2.2) 


\section{Euratom PREDIS}

\section{WP7-T7.3: Testing and Monitoring}

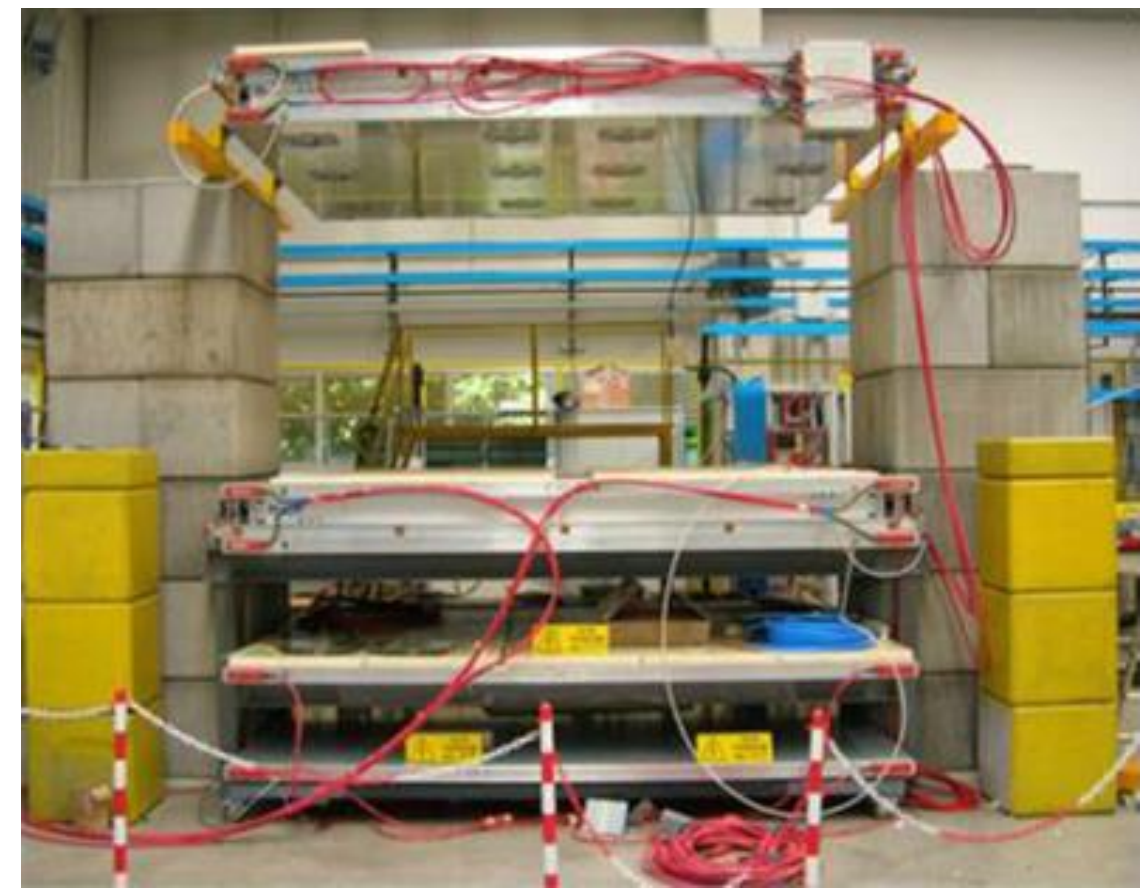

Our largest tool:

Muon Tomography

\section{INFN}




\section{Euratom PREDIS}

\section{WP7-T7.3: Testing and Monitoring}

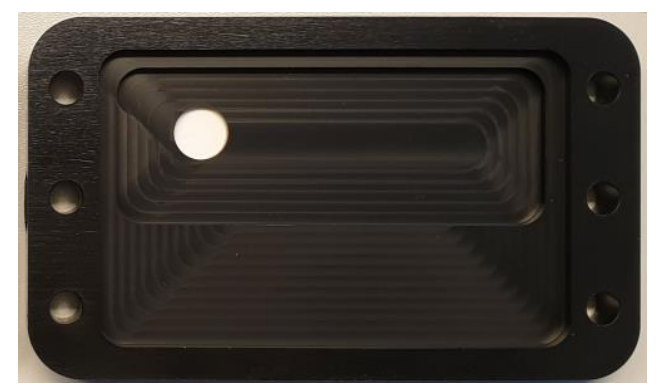

Our smallest tools:
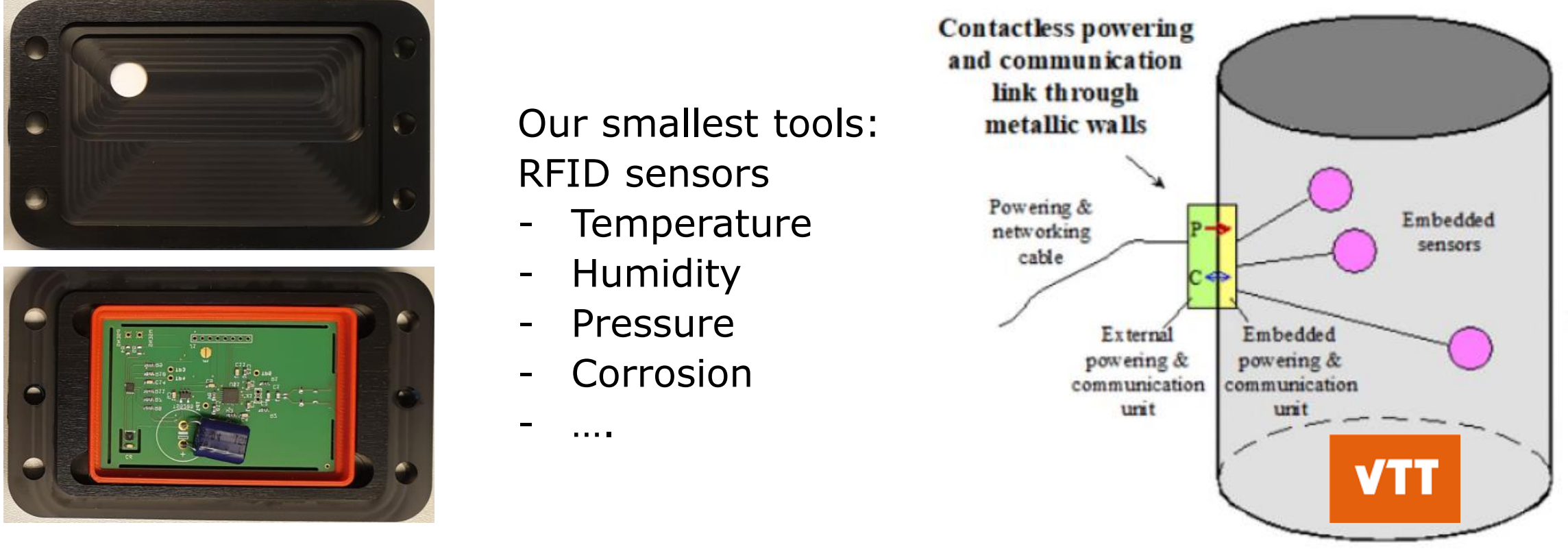

RFID sensors

- Temperature

- Humidity

- Pressure

- Corrosion

- 


\section{Euratom PREDIS}

\section{WP7-T7.4: Digital Twin}

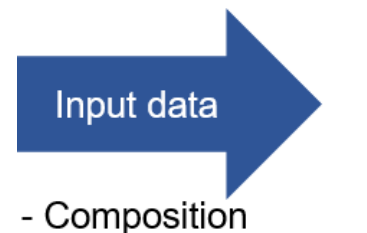

(cement, aggregates, waste,...)

- Design, geometry

- Storage conditions

(Monitoring data)

Models for:

moisture evolution,

carbonation, ASR, gas

generation, metal

corrosion, swelling

reactions etc.

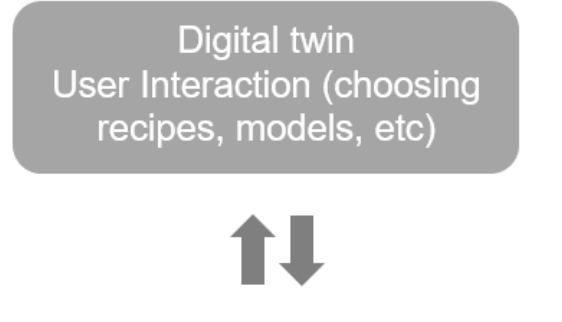

Digital twin

(summary computer model of waste package integrity evolution, surrogate models)

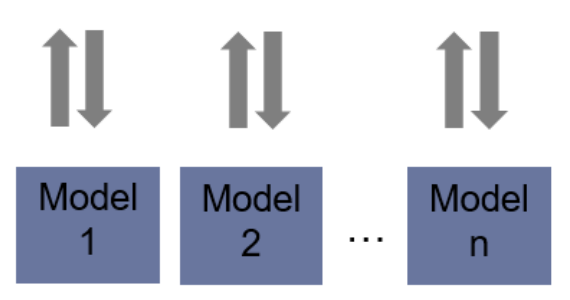

Calibration,

Predicted integrity evolution

- Gas production

- Volume expansion

- Package corrosion

- $\ldots . .$.

samples,...

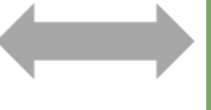
validation

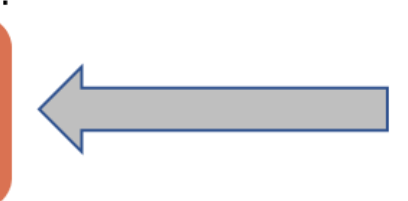

Data from

characterization

of old cemented

waste

packages,

old cement

Decision

framework

(dashboard),

decisions for

the user

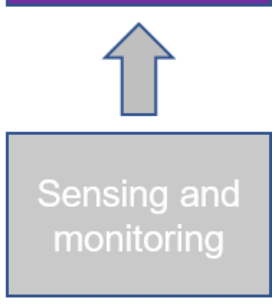

Sensing and
monitoring 


\section{Euratom PREDIS}

\section{WP7-T7.5: Data/Decision Framework}
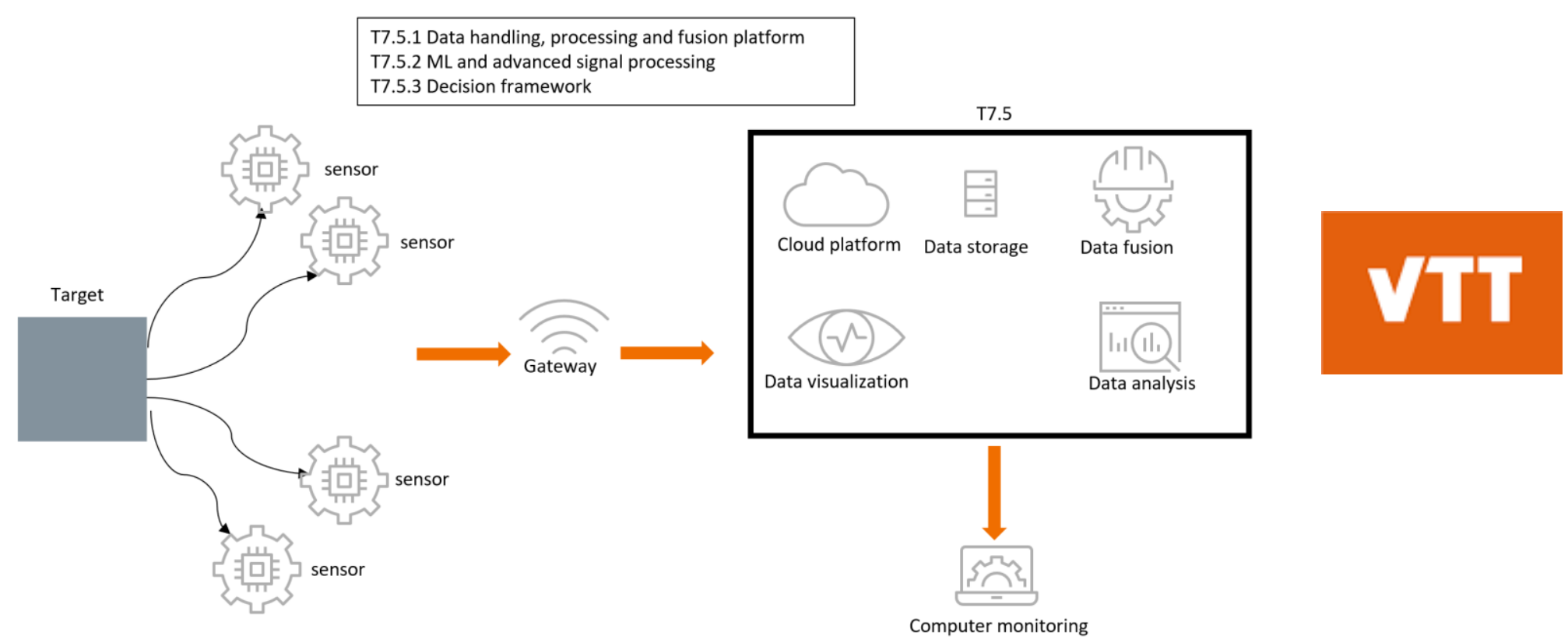

Computer monitoring 


\section{Euratom PREDIS \\ WP7-T7.6: Demonstration}

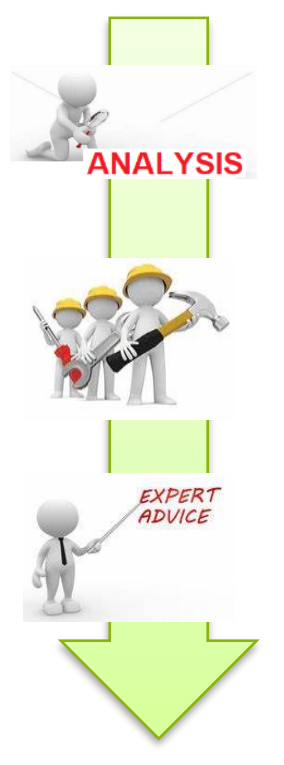

- Subtask T7.6.1 Evaluation of technologies and developed systems from an end-user perspective

- Develop a waste package prototype for performing large-scale trials,

- choose and evaluate the most relevant and promising NDE/sensing techniques

- Subtask T7.6.2 Demonstrating systems and methods

- Implement the experimental set-up defined in Subtask 6.1,

- Select the technologies to be validated,

- perform a series of full-scale trials in a realistic testing environment

- Subtask T7.6.3 Definition of potential mitigation actions and design improvements

- Proposal of improved designs that eliminate any weak points identified during the course of the project and conceptual design for the use of the project results in automatized store concepts 


\section{Euratom PREDIS \\ WP7: Impact}

More versatile and reliable condition monitoring technologies, which have been demonstrated on operating radioactive facilities and made available to end users

Improved accuracy in predicting the behaviour of waste/packages in stores through the integration of models with store and package monitoring information obtained using digital and machine learning technologies to enhance sampling, monitoring strategies and multi-method data fusion

Increased safety: reduction of exposure time to personnel connected to remediation activities, reduction of risk of RN dispersion (locally or to the environment), gaining local stakeholder trust

Reduced cost ( $20 \%$ or greater reduction in costs related to late-stage detection of damage or deterioration within waste packages)

Minimised environmental footprint resulting from optimised treatment, packaging and store operations. 


\section{Euratom PREDIS}

\section{WP7: Deliverables}

All 10 deliverables in WP7 are of the type "public" and will be made available to anybody interested in the project and its results.

Reports and publications can be downloaded from the PREDIS project website. Topics covered will be:

- innovative integrity testing and monitoring techniques and its demonstration

- digital twin and modelling technologies

- innovative data handling and decision framework technologies

- report on the economic, environmental, and safety impact

The State of the Art report is already available, other reports/articles will be published in $2023 \& 2024$ 


\section{Euratom PREDIS \\ Events}

Stakeholder free on-line webinar series continues in autumn 2021, addressing industry needs in pre-diposal management:

- 26.10. at 9-12 CET on PREDIS developments of geopolymers for pre-disposal waste management (WP4-6 issues)

- 25.11. at 9-12 CET on PREDIS State of Knowledge (WP3 - covering knowledge documents, training, mobility activities and opportunities)

- Q1/2022: Digital Twin technology (WP7)

- 25-27.4.2022, PREDIS Annual Workshop, Berlin, Germany

- 18-20.1.2023, International Conference on Non-destructive Evaluation of Concrete in Nuclear Application, Espoo, Finland 